Artigo / Article

\title{
Tratamento do Mieloma Múltiplo recidivado
}

\section{Relapsed Multiple Myeloma treatment}

Vania T. M. Hungria

\section{Introdução}

Apesar dos progressos no tratamento do mieloma múltiplo, com aumento da sobrevida livre de progressão observada principalmente em pacientes abaixo de 65 anos, a maioria dos pacientes recidivará.

O padrão da recidiva é muito heterogêneo, podendo evoluir com comportamento indolente ou agressivo. Enquanto alguns pacientes apresentam recidiva precoce, com pior prognóstico, e provavelmente responderão mal ao tratamento, outros apresentam recidiva após um longo período de plateau e provavelmente atingirão uma melhor resposta à terapia.

O tratamento da doença recidivada depende de vári- os fatores: do tratamento realizado como primeira linha, se transplante autólogo de medula óssea ou não, da resposta e sua duração, se a recidiva ocorreu com ou sem tratamento de manutenção, do performance status do paciente e da reserva medular. ${ }^{1} \mathrm{Na}$ maioria dos casos, o objetivo terapêutico será controlar a doença, melhorar os sintomas e a qualidade de vida. $^{2}$

\section{Quando tratar a recidiva?}

Os pacientes que apresentam recidiva indolente, com aumento assintomático dos níveis séricos do componente monoclonal, isto é, sem anemia ou aumento da creatinina ou hipercalcemia, ou evolução da doença óssea, podem ter seu

Professora adjunta da Disciplina de Hematologia e Oncologia da Faculdade de Ciências Médicas da Santa Casa de São Paulo.

Correspondência: Vania Tietsche de Moraes Hungria

Rua Marquês de Itu, 579 - $3^{\circ}$ andar

01223-000 - São Paulo-SP - Brasil

Fone: (11) 2176-7293

E-mail: hungria@dialdata.com.br 
tratamento adiado para quando se apresentarem sintomáticos. Já os pacientes sintomáticos, com recidiva agressiva, devem ser tratados imediatamente.

\section{Quais as opções de tratamento na recidiva?}

- Repetir a terapia inicial.

- Altas doses de quimioterapia.

- VAD (vincristina, doxorrubicina e dexametasona) ou esquemas similares.

- MP (melfalano e prednisona), VBMCP (vincristina, carmustina, melfalano, ciclofosfamida e prednisona), ou esquemas similares.

- Altas doses de dexametasona.

- Talidomida como monoterapia ou associada a outras drogas.

- Bortezomibe como monoterapia ou associado a outras drogas.

- Lenalidomida (não aprovada no Brasil) como monoterapia ou associada a outras drogas.

- Novos agentes em estudos clínicos.

- Novas combinações de drogas já utilizadas.

\section{Como decidir entre as opções?}

Considerando que não há terapia curativa para mieloma múltiplo, todas as opções de tratamento devem ser testadas. Não há nenhum resultado definitivo quanto à melhor seqüência de tratamento.

Pacientes que recidivam fora dos esquemas de tratamento utilizados inicialmente podem atingir uma segunda remissão com o mesmo esquema ou similares. Se a recidiva ocorrer por mais de seis meses do término da terapia convencional, o esquema quimioterápico deverá ser reinstituído.

Os pacientes que foram inicialmente tratados com esquema MP e atingiram resposta, se repetido o esquema na recidiva, 50\% destes poderão alcançar resposta novamente. ${ }^{3}$

Os pacientes que não respondem aos agentes alquilantes deveriam receber esquema VAD (vincristina, doxorrubicina, dexametasona) ou altas doses de dexametasona. ${ }^{4}$

O transplante autólogo de medula óssea pode ser considerado em pacientes que não realizaram o transplante como terapia inicial, se as células-tronco periféricas tiverem sido coletadas anteriormente. ${ }^{5,6}$ Um segundo transplante pode também ser eficaz em pacientes que recidivam após o transplante inicial.

Pacientes com mieloma múltiplo recidivado que têm pelo menos um dos seguintes fatores favoráveis: baixos valores de $\beta_{2}$ microglobulina no resgate, apenas um transplante, e recidiva ocorrendo acima de 12 meses, podem ter uma sobrevida projetada aos 18 meses de 79\%, comparado com 38\% para pacientes com nenhuma destas variáveis favoráveis. $^{7,8}$

Não há estudos comparando um segundo transplante autólogo com outras estratégias na recidiva. Não há dados suficientes na literatura para recomendar o transplante alogênico como terapia de resgate para pacientes com mieloma múltiplo recidivado.

Nos últimos cinco anos, grandes avanços têm ocorrido com a utilização da talidomida ${ }^{9-19}$ e o aparecimento de novas abordagens terapêuticas, como o bortezomibe..$^{27-51}$

\section{Talidomida}

A descoberta da angiogênese aumentada em mieloma múltiplo, junto com o reconhecimento das propriedades antiagiogênicas da talidomida levaram ao primeiro estudo clínico com esta droga na Universidade de Arkansas. ${ }^{9}$ Os pacientes receberam inicialmente a dose de $200 \mathrm{mg} /$ dia, e as doses foram aumentadas em $200 \mathrm{mg}$ a cada duas semanas, até atingir a dose de $800 \mathrm{mg} /$ dia. Na avaliação de 169 pacientes, pré-tratados (pesadamente), a redução do componente monoclonal (maior que 50\%) foi observada em 30\% dos pacientes. A sobrevida livre de evento aos dois anos foi de $20 \%$ e a sobrevida global, de $48 \% .{ }^{10}$ Estes resultados foram impressionantes porque 2/3 dos pacientes incluídos neste estudo tinham falhado ao duplo transplante autólogo da medula óssea.

Desde então, vários estudos têm demonstrado que a talidomida produz taxas de resposta em aproximadamente 25\% a 35\% dos pacientes com mieloma múltiplo recidivado e refratário. ${ }^{11-14} \mathrm{O}$ tempo mediano para se obter a resposta é de um a dois meses. A duração mediana da resposta é de aproximadamente 12 meses. A dose ótima da talidomida não está estabelecida, mas é geralmente utilizada na dose de $200 \mathrm{mg} /$ dia. A dose da talidomida deve ser individualizada e, após a resposta ter sido atingida, a dose deve ser ajustada para a menor dose que se possa alcançar e manter uma resposta, para minimizar a toxicidade a longo prazo.

Dada a atividade da talidomida como um agente único em mieloma múltiplo, estudos subseqüentes pesquisaram o seu uso em combinação com outros agentes ativos no tratamento do mieloma recidivado.

Devido ao sinergismo de ação in vitro com dexametasona, a avaliação da combinação de talidomida e dexametasona foi obrigatória. A taxa de resposta, quando a talidomida foi usada com corticoesteróides, comparada com a taxa da talidomida como agente único, aumentou para aproximadamente $50 \%$. $^{15-17}$

Embora a taxa de resposta desta associação tenha aumentado em até $20 \%$, a superioridade da talidomida/dexametasona versus talidomida sozinha não foi superior em ter- 
mos de sobrevida livre de progressão de doença. Entretanto, esta combinação pode ser superior à quimioterapia convencional. ${ }^{11}$

A combinação de talidomida, dexametasona e ciclofosfamida (CTD) pode aumentar a taxa de resposta para $65 \% .{ }^{18,19}$ Várias outras combinações quimioterápicas contendo a talidomida estão sendo estudadas, incluindo a dexametasona, talidomida, cisplatina, doxorubicina, ciclofosfamida e etoposide (DT-PACE) ${ }^{20}$ claritromicina, talidomida em dose baixa e dexametasona (BLT-D), ${ }^{21}$ e melfalano, talidomida e dexametasona (MTD). ${ }^{22}$

\section{Efeitos adversos}

Os efeitos colaterais mais freqüentes da talidomida são: sonolência, fadiga, constipação e exantema, mas geralmente melhoram com a redução da dose. ${ }^{23}$ A neuropatia periférica ocorre com o uso a longo prazo e freqüentemente necessita a descontinuação da terapia ou redução da dose. A incidência da trombose venosa profunda é de apenas $1 \%$ a $3 \%$ em pacientes que recebem a talidomida como monoterapia, mas aumenta para $10 \%$ a $15 \%$ nos pacientes que a recebem em combinação com a dexametasona, e para aproximadamente $25 \%$ nos pacientes que recebem a droga em combinação com outros agentes quimioterápicos citotóxicos, especialmente a doxorubicina. ${ }^{10,24-26}$ Outros efeitos adversos incluem: edema, bradicardia, neutropenia, impotência e o hipotiroidismo.

\section{Bortezomibe}

O bortezomibe foi o primeiro inibidor de proteassoma a ser testado em estudos clínicos. O efeito terapêutico da inibição do proteassoma induzido pelo bortezomibe no mieloma é, provavelmente, resultado da citotoxicidade direta e dos efeitos no microambiente da medula óssea. ${ }^{27,28}$ Uma das conseqüências da inibição do proteassoma é o acúmulo do inibidor kappa B (I КB), um inibidor do fator de

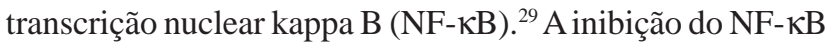
leva a uma diminuição da expressão das moléculas de adesão e de vários fatores de proliferação, sobrevida e angiogênicos. Causa também uma diminuição das proteínas que

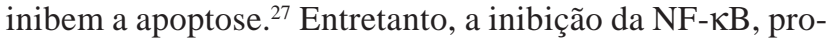
vavelmente, não é o único mecanismo de efeito antimieloma. ${ }^{30}$

\section{Estudos clínicos}

A eficácia promissora contra o mieloma foi observada em estudo fase I. ${ }^{31}$ Com base nesta observação, um estudo multicêntrico fase II (SUMMIT), para pacientes com mieloma múltiplo recidivado foi iniciado, utilizando o bortezomibe na dose de $1,3 \mathrm{mg} / \mathrm{m}^{2}$, em infusão intravenosa de três a cinco segundos nos dias 1, 4, 8 e 11, em ciclos de 21 dias, por no máximo oito ciclos, e a dexametasona foi associada em pacientes com pouca resposta ao bortezomibe isola- do. ${ }^{32}$ Dos 193 pacientes que foram avaliados, 92\% tinham recebido três ou mais das principais classes de drogas antimieloma e, em 91\%, a doença tinha sido refratária ao último tratamento. A taxa de resposta completa e parcial com bortezomibe foi de $27 \%$, sendo que $10 \%$ dos pacientes atingiram resposta completa ou próximo da resposta completa. A duração mediana da resposta foi de 12 meses e as respostas foram associadas à melhora da citopenia, função renal e qualidade de vida. ${ }^{33}$

Em outro estudo fase II (CREST), os pacientes com mieloma múltiplo recidivado foram aleatoriamente designados para receber uma de duas doses de bortezomibe (28 pacientes foram designados a receber uma dose de $1,0 \mathrm{mg} / \mathrm{m}^{2} \mathrm{e}$ 26 pacientes, 1,3 mg $/ \mathrm{m}^{2}$ ) nos dias 1, 4, 8 e 11 em um ciclo de 21 dias por um total de oito ciclos. As respostas ocorreram em $33 \%$ dos que receberam $1,0 \mathrm{mg} / \mathrm{m}^{2}$ e em $50 \%$ dos que recebe$\operatorname{ram} 1,3 \mathrm{mg} / \mathrm{m}^{2} .{ }^{34}$

Nos dois estudos fase II (SUMMITT e CREST), a adição da dexametasona para os pacientes que apresentaram pouca resposta resultou em aumento da taxa de resposta para $18 \%$ e $33 \%$, respectivamente. ${ }^{35}$

Apesar dos estudos iniciais permitirem um máximo de apenas oito ciclos de bortezomibe, dados recentes indicam que é seguro ministrar pelo menos cinco ou seis ciclos adicionais de tratamento com efeitos colaterais controláveis. ${ }^{36}$

O estudo APEX, realizado em 95 centros, foi um estudo fase III, randomizado, comparando bortezomibe com dexametasona. Foram incluídos 669 pacientes com mieloma múltiplo recidivado após uma a três linhas de terapia. Este estudo mostrou que o bortezomibe é mais eficaz do que as altas doses de dexametasona, demonstrado pelo aumento significante da taxa de resposta ( $43 \%$ vs $18 \%$ ), tempo mediano para progressão de doença (6,2 vs 3,4 meses) e taxa de sobrevida de um ano ( $80 \%$ vs $67 \%$, respectivamente). Um dado muito importante foi com relação ao tempo para início da resposta, que foi freqüentemente muito rápido, após um a dois ciclos. ${ }^{37,38}$

Numa subanálise do estudo APEX, a atividade do bortezomibe foi especificamente analisada em pacientes idosos, e a resposta foi igualmente eficaz e bem tolerada no grupo de jovens e idosos. Outros fatores prognósticos desfavoráveis, como ß2 microglobulina elevada e refratariedade aos tratamentos anteriores, não influenciaram nas taxas de resposta e no tempo para progressão com bortezomibe. ${ }^{39}$

Ainda com relação aos fatores de alto risco, a deleção do cromossomo 13 está associada a baixas taxas de resposta e sobrevida mais curta. Numa subanálise do estudo APEX, no grupo de pacientes que receberam dexametasona, a deleção do cromossomo 13 foi associada com menor sobrevida; enquanto que os pacientes que receberam bortezomibe, a deleção do cromossomo 13 não teve impacto na sobrevida. ${ }^{40}$ 
Outros estudos clínicos que avaliaram os resultados do tratamento com bortezomibe para pacientes com mieloma múltiplo recidivado e pacientes com mieloma múltiplo recém-diagnosticados, também demonstraram que as taxas de resposta não foram influenciadas por anormalidades citogenéticas. ${ }^{41,42}$

Um achado interessante é que o bortezomibe é altamente eficaz em plasmocitomas extramedulares. ${ }^{43}$

Embora estes resultados sejam animadores, uma proporção de pacientes não responde ao bortezomibe, e a resistência adquirida já tem sido observada. Estes fatos, junto ao comprovado sinergismo in vitro do bortezomibe com outras drogas, justificam a combinação da quimioterapia.

Estudos pilotos, incluindo pacientes com mieloma recidivado, associando o bortezomibe com melfalano ${ }^{39} \mathrm{ou}$ doxorrubicina lipossomal ${ }^{40}$ ou ciclofosfamida e dexametasona ${ }^{41}$ demonstraram taxas de resposta de $50 \%$ a $76 \%$.

A associação de bortezomibe e talidomida também tem sido testada. Em uma série de 56 pacientes com mieloma múltiplo refratário, a taxa de resposta global foi de $70 \%$, com 22\% de resposta completa e próximo de resposta completa, sem aumentar a toxicidade com relação a neuropatia e mielossupressão..$^{42}$ Outra combinação de talidomida e bortezomibe, com adriamicina e dexametasona, também tem sido avaliada, com taxa de resposta completa e parcial de $55 \%$ e toxicidade aceitável. ${ }^{43}$

As respostas destas combinações são superiores àquelas obtidas com bortezomibe sozinho, confirmando o efeito sinérgico encontrado em estudos in vitro.

\section{Efeitos adversos}

Os efeitos colaterais mais freqüentes do bortezomibe foram os sintomas gastrintestinais, fadiga e anorexia, na maioria das vezes grau 1 e $2 .{ }^{46}$ A plaquetopenia grau 3 e 4 , devido ao bloqueio reversível na liberação das plaquetas pelos megacariócitos, foi encontrada em $30 \%$ dos casos, enquanto a anemia e neutropenia foram raras $(<10 \%))^{32,34,35,37,49} \mathrm{O}$ efeito colateral mais incômodo é a neuropatia periférica sensitiva e dor neuropática (37\%, com 9\% grau 3), embora este efeito possa ser resolvido ou melhorado em $2 / 3$ dos pacientes após completar ou descontinuar a terapia. É importante a atenção aos sintomas de neuropatia, pois a redução da dose, de acordo com as recomendações estabelecidas, ajuda a evitar a piora dos sintomas e a necessidade de interromper o tratamento. ${ }^{32,34,35,37,49-51}$

\section{Conclusão}

Nos últimos 40 anos, os esquemas para tratamento dos pacientes com mieloma múltiplo vêm melhorando. Nos anos 80 , com a introdução das altas doses de quimioterapia levando a remissão completa em uma proporção significante de pacientes, obtivemos uma esperança de controle da doença mais prolongado. Na década de 90 , a disponibilidade de drogas antimieloma com novos mecanismos de ação e eficazes, têm trazido melhores expectativas com relação aos resultados do tratamento do mieloma.

Apesar dos progressos no tratamento, o mieloma múltiplo ainda é uma doença incurável, e a maioria dos pacientes recidivará.

Há várias opções de tratamento para o paciente com mieloma múltiplo recidivado, e a decisão dependerá do padrão da recidiva, do tratamento realizado como primeira linha, se transplante autólogo de medula óssea ou não, da resposta e sua duração, se a recidiva ocorreu com ou sem tratamento de manutenção, do performance status do paciente e da reserva medular.

Dentre as opções terapêuticas, podem ser considerados a reintrodução do tratamento utilizado como terapia inicial, o transplante autólogo de medula óssea e as novas drogas, como a talidomida e o bortezomibe, drogas disponíveis para uso no Brasil. A experiência clínica com estas drogas em pacientes com mieloma múltiplo recidivado é extensa, tanto como monoterapia ou em combinações quimioterápicas. Os efeitos colaterais estão bem definidos e a maioria destes é controlável.

\section{Recomendações para o tratamento do mieloma múltiplo recidivado}

- $\quad$ O tratamento deve ser individualizado dependendo da idade, da função da medula óssea, do tratamento inicial, do tempo e padrão da recidiva.

- A maioria dos pacientes tratados com melfalano e prednisona que atingiram remissão, quando recidiva após seis meses do final do tratamento, respondem ao mesmo esquema.

- Transplante autólogo de medula óssea pode ser considerado em pacientes que não tenham realizado este procedimento como terapia inicial, se as células tronco-periféricas tiverem sido coletadas anteriormente.

- $\quad$ Um segundo transplante autólogo pode ser eficaz, especialmente nas recidivas após pelo menos 12 a 18 meses.

- $\quad$ Talidomida como monoterapia ou em combinação com outras drogas. Pacientes que utilizaram talidomida como tratamento inicial ou manutenção, podem responder às associações com outros agentes.

- $\quad$ Bortezomibe está indicado como monoterapia ou associado a dexametasona e a outros quimioterápicos. 


\begin{abstract}
Multiple myeloma still remains an incurable disease. Despite the new treatment approaches, almost all patients face the risk of an eventual relapse. The pattern of relapse is very heterogeneous and can be indolent or more aggressive. The treatment of relapsed disease depends on a number of factors: duration of response, relapse on or off maintenance therapy, prior therapy and specially prior autologous stem cell transplantation, performance status, hematopoietic reserve. If relapse occurs more than 6 months after therapy ended, the initial chemotherapy regimen should be reinstituted. Autologous stem cell transplantation can be proposed as consolidation therapy in chemosensitive relapses or as salvage therapy if stem cells have been collected earlier. Thalidomide has been tested in relapsed multiple myeloma and is now considered as standard treatment for patients relapsing after conventional chemotherapy or after autologous stem cell transplantation. Thalidomide alone can induce objective responses in at least onethird of heavily pretreated patients and, combined with chemotherapy, objective responses can be achieved by two-thirds of the patients. Bortezomib is indicated, alone or in combination with other agents, for relapsed patients and can produce an overall response rate of $43 \%$ to $76 \%$. The most appropriate management must be individualized depending on the age, bone marrow function, prior therapy and the timing of the relapse. Rev. bras. hematol. hemoter. 2007;29(1):48-53.
\end{abstract}

Key words: Relapsed multiple myeloma; thalidomide; bortezomib.

\section{Referências Bibliográficas}

1. Harousseau JL. Management of multiple myeloma. Rev Clin Exp Hematol 2002;6:253-275.

2. Smith A, Wisloff F, Samson D. Guidelines on the diagnosis and management of multiple myeloma 2005. Br J Haematol 2006; 132:410-451.

3. Belch A, Shelley W, Bergsagel D, et al. A randomized trial of maintenance vs. no maintenance melphalan and prednisone in responding multiple myeloma patients. British Journal of Cancer 1988;57:94-99.

4. Barlogie B, Smith L, Alexanian R. Effective treatment of advanced multiple myeloma refractory to alkylating agents. N Engl J Med 1984;310:1.353-1.356.

5. Gertz MA, Lacy MQ, Inwards DJ, et al. Early harvest and late transplantation as an effective therapeutic strategy in multiple myeloma. Bone Marrow Transplant 1999;23:221-226.

6. Fermand JP, Ravaud P, Chevret S, et al. High-dose therapy and autologous peripheral blood stem cell transplantation in multiple myeloma: up-front or rescue treatment? Blood 1998;92:3.1313.136 .

7. Tricot G, Jagannath S, Vesole DH, et al. Relapse of multiple myeloma after autologous transplantation: survival after salvage therapy. Bone Marrow Transplantation 1995;16:7-11.

8. Mehta J, Tricot G, Jagannath S, et al. Salvage autologous or allogeneic transplantation for multiple myeloma refractory to or relapsing after a first line autograft. Bone Marrow Transplantation 1998;21:887-898.

9. Singhal S, Mehta J, Desikan R, et al. Antitumor activity of thalidomide in refractory multiple myeloma. N Engl J Med 1999; 341:1.565-1.571.
10. Barlogie B, Desikan R, Eddlemon P, et al. Extended survival in advanced and refractory multiple myeloma aftr single-agent thalidomide: identification of prognostic factors in a phase 2 study of 169 patients. Blood 2001;98:492-494.

11. Dimopoulos M, Anagnostopoulos A, Weber D, et al. Treatment of plasma cell dyscrasias with thalidomide and its derivatives. J Clin Oncol 2003;21:4.444-4.454.

12. Kumar S, Witzig TE, Rajkumar SV. Thalidomide: current role in the treatment of non-plasma cell malignancies. J Clin Oncol $2004 ; 22: 2.477-2.488$.

13. Richardson P, Schlossman R, Jagannath S, et al. Thalidomide for patients with relapsed multiple myeloma after high-dose chemotherapy and stem cell transplantation: results of an openlabel multicenter phase 2 study of efficacy, toxicity, and biological activity. Mayo Clin Proc 2004;79:875-882.

14. Kumar S, Gertz MA, Dispenzieri A, et al. Response rate, durability of response, and survival after thalidomide therapy for relapsed multiple myeloma. Mayo Clinic Proc 2003;78:34-39.

15. Dimopoulos MA, Zervas K, Kouvatseas G, et al. Thalidomide and dexamethasone combination for refractory multiple myeloma. Ann Oncol 2001;12:991-995.

16. Palumbo A, Giaccone L, Bertola A, et al. Low-dose thalidomide plus dexamethasone is an effective salvage therapy for advanced myeloma. Haematologia 2001;86:399-403.

17. Anagnostopoulos A, Weber D, Rankin K, et al. Thalidomide and dexamethasone for resistant multiple myeloma. Br J Haematol 2003;121:768-771.

18. Garcia-Sanz R, Gonzalez-Fraile MI, Sierra M, Lopez C, et al. The combination of thalidomide, cyclophosphamide and dexamethasone (ThaCyDex) is feasible and can be an option for relapsed/refractory multiple myeloma. Hematol J 2002; 3:43-48.

19. Dimopoulos MA, Hamilos G, Zomas A, et al. Pulsed cyclophosphamide, thalidomide and dexamethasone: an oral regimen for previously treated patients with multiple myeloma. Hematol J 2004;5:112-117.

20. Barlogie B, Shaughnessy J, Tricot G, et al. Treatment of multiple myeloma. Blood 2004;103:20-32.

21. Coleman M, Leonard J, Lyons L, et al. BLT-D (clarithromycin (Biaxin), low-dose thalidomide, and dexamethasone) for the treatment of myeloma and Waldenstrom's macroglobulinemia. Leuk Lymphoma 2002;43:1.777-1.782.

22. Srkalovic G, Elson P, Trebisky B, et al. Use of melphalan, thalidomide, and dexamethasone in treatment of refractory and relapsed multiple myeloma. Med Oncol 2002;19:219-226.

23. Ghobrial IM, Rajkumar SV. Management of thalidomide toxicity. J Supportive Oncol 2003;344:1.951-1.952.

24. Zangari M, Anaissie E, Barlogie B, et al. Increased risk of deepvein thrombosis in patients with multiple myeloma receiving thalidomide and chemotherapy. Blood 2001:98:1.614-1.615.

25. Zangari M, Siege E, Barlogie B, et al. Thrombogenic activity of doxorubicin in myeloma patients receiving thalidomide: implications for therapy. Blood 2002;100:1.168-1.171.

26. Osman K, Comenzo R, Rajkumar SV. Deep venous thrombosis and thalidomide therapy for multiple myeloma. N Engl J Med 2001; 344:1.951-1.952.

27. Mitsiades N, Mitsiades CS, Poulaki V, et al. Molecular sequelae of proteasome inhibition in human multiple myeloma cells. Proc Natl Acad Sci USA 2002;99:14.374-14.379.

28. Hideshima T, Mitsiades C, Akiyama M, et al. Molecular cechanisms mediating anti-myeloma activity of proteasome inhibitor PS-341. Blood 2003;101:1.530-1.534.

29. Palombella VJ, Rando OJ, Goldberg AL, Maniatis T. The ubiquitinproteasome pathway is required for processing the NF-kB1 precursor protein and the activation of NF-kB. Cell 1994;78:773-785. 
30. Hideshima T, Richardson P, Chauhan D, et al. The proteasome inhibitor PS-341 inhibits growth, induces apoptosis, and overcomes drug resistance in human multiple myeloma cells. Cancer Res 2001; 61:3.071-3076.

31. Orlowski RZ, Stinchcombe TE, Mitchell BS, et al. Phase I trial of the proteasome inhibitor PS-341 in patients with refractory hematologic malignancies. J Clin Oncol 2002; 20: 4.420-4.427.

32. Richardson P, Barlogie B, Berenson J, et al. A phase 2 study of bortezomib in relapsed, refractory myeloma. N Engl J Med 2003; 348:2.609-2.617.

33. Lee A, Richardson PG, Barlogie B, et al. Quality-of-life (QOL) and clinical benefit assessment in patients with relapsed and refractory multiple myeloma (MM) treated with bortezomib. Prog Proc Am Soc Clin Oncol 2003;22:582 (abstract).

34. Jagannath S, Barlogie B, Berenson J, et al. A phase 2 study of two doses of bortezomib in relapsed or refractory myeloma. $\mathrm{Br} \mathrm{J}$ Haematol 2004;127:165-172.

35. Jagannath S, Richardson P, Barlogie B, et al. Bortezomib in combination with dexamethasone for the treatment of patients with relapsed and or refractory multiple myeloma with less than optimal response to bortezomib alone. Haematologica 2006;91: 929-934.

36. Berenson JR, Jagannath S, Barlogie B, et al. Safety of prolonged therapy with bortezomib in relapsed or refractory multiple myeloma. Cancer 2005;104:2.141-2.148.

37. Richardson PG, Sonneveld P, Schuster MW, et al. Bortezomib or high dose dexamethasone for relapsed multiple myeloma. N Engl J Med 2005;352:2.487-2.498.

38. Richardson P, Sonneveld P, Schuster M, et al. Bortezomib continues demonstrates superior efficacy compared with high-dose dexamethasone in relapsed multiple myeloma: updated results of the APEX trial. Blood 2005;106:2.547 (abstract).

39. Richardson PG, Sonneveld P, Schuster MW, et al. Safety and efficacy of bortezomib in high-risk and elderly patients with relapsed myeloma. J Clin Oncol 2005;23:568S (Abstract 6533).

40. Jagannath S, Richardson PG, Sonneveld P, et al. Bortezomib appears overcome the poor prognostic impact of chromosome 13 deletion in phase 2 and 3 clinical studies. Haematologica 2005; 90 (Suppl2): 248 (Abstract 620).

41. Drach J, Kuenburg E, Sagaster V, et al. Short survival, despite promising response rates, after bortezomib treatment of multiple myeloma patients with a 13q-deletion. Blood 2005; 106 (Abstract 509).

42. Mateos MV, Hernandez JM, Herandez MT, et al. Bortezomib plus melphalan and prednisone in elderly untreated patients with multiple myeloma: results of a multicenter phase 1/1 study. Blood 2006;108:2.165-2.172.

43. Rosiñol L, Cibeira MT, Uriburu C, et al. Bortezomib: an effective agent in extramedullary disease in multiple myeloma. Eur J Haematol 2006;76:405-408.

44. Berenson JR, Yang HH, Sadler K, et al. Phase I/II trial assessing bortezomib and melphalan combination therapy for the treatment of patients with relapsed or refractory multiple myeloma J Clin Oncol 2006;24:937-944.

45. Orlowski RZ, Voorhees PM, Garcia RA, et al. Phase 1 trial of the proteasome inhibitor bortezomib and pegylated liposomal doxorubicin in patients with advanced hematologic malignancies. Blood 2005;105:3.058-3.065.

46. Kropff M, Bisping G, Liebisch B. Bortezomib in combination with high-dose dexamethasone and continuous low-dose oral cyclophosphamide for relapsed multiple myeloma. Blood 2005; 106:2.549 (abstract).

47. Zangari M, Barlogie B, Burns M. Velcade (V) -Thalidomide (T) dexamethasone (D) for advanced and refractory multiple myeloma
(MM): long-term follow-up of phase I-II Trial UARK 2001-37: superior outcome in patients with normal cytogenetics and no prior T. Blood 2005;106:2.552 (abstract).

48. Chanan-Khan A, Miller KC. Velcade, doxil and thalidomide (VDT) is an effective salvage regimen for patients with relapsed and refractory multiple myeloma. Leuk Lymphoma 2005;46:1.1031.104.

49. San Miguel J, Bladé J, Boccadoro M, et al. A practical update on the use of bortezomib in the management of multiple myeloma. Oncologist 2006;11:51-61.

50. San Miguel J, Richardson P, Sonneveld P. Frequency, characteristics, and reversibility of peripheral neuropathy (PN) in the APEX Trial. Blood 2005;106:366 (abstract).

51. Richardson P, Briemberg H, Jagannath S, et al. Frequency, characteristics, and reversibility of peripheral neuropathy during treatment of advanced multiple myeloma with bortezomib. J Clin Oncol 2006;24:3.113-3.120.

O tema apresentado e o convite ao(s) autor(es) consta da pauta elaborada pelo co-editor.

Avaliação: Co-editor e um revisor externo. Publicado após revisão e concordância do editor. Conflito de interesse: não declarado.

Recebido: 25/11/2006

Aceito: 05/01/2007 